\title{
Alcohol Needs Assessment Research Project (ANARP). The 2004 National Alcohol Needs Assessment for England
}

Link to publication record in Manchester Research Explorer

\section{Citation for published version (APA):}

Drummond, C., Oyefeso, A., Phillips, T., Cheeta, S., Deluca, P., \& Perryman, K. (2005). Alcohol Needs

Assessment Research Project (ANARP). The 2004 National Alcohol Needs Assessment for England. Department of Health and Social Care.

http://webarchive.nationalarchives.gov.uk/20130107105354/http://dh.gov.uk/en/publicationsandstatistics/publicatio ns/publicationspolicyandguidance/dh_4122341

\section{Citing this paper}

Please note that where the full-text provided on Manchester Research Explorer is the Author Accepted Manuscript or Proof version this may differ from the final Published version. If citing, it is advised that you check and use the publisher's definitive version.

\section{General rights}

Copyright and moral rights for the publications made accessible in the Research Explorer are retained by the authors and/or other copyright owners and it is a condition of accessing publications that users recognise and abide by the legal requirements associated with these rights.

\section{Takedown policy}

If you believe that this document breaches copyright please refer to the University of Manchester's Takedown Procedures [http://man.ac.uk/04Y6Bo] or contact uml.scholarlycommunications@manchester.ac.uk providing relevant details, so we can investigate your claim.

\section{OPEN ACCESS}




\section{(DH) Department
of Health}

\section{Alcohol Needs Assessment Research Project (ANARP)}

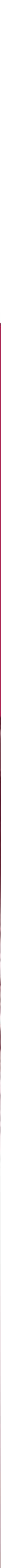




\section{Alcohol Needs Assessment Research Project (ANARP) \\ The 2004 national alcohol needs assessment for England}

Colin Drummond MB ChB MD FRCPsych ${ }^{\mathrm{a} 1}$, Adenekan Oyefeso PhD ${ }^{\mathrm{a}}$, Tom Phillips MSc RMN ${ }^{\mathrm{a}}$, Survjit Cheeta PhD ${ }^{a}$, Paolo Deluca $\mathrm{PhD}^{\mathrm{a}}$, Katherine Perryman MSc${ }^{a}$, Hannah Winfield $\mathrm{BA}^{\mathrm{a}}$, Jennie Jenner BSca, Kathryn Cobain MSc ${ }^{a}$, Sue Galea MD MRCPsycha, Vivienne Saunders MSc ${ }^{a}$, Tom Fuller ${ }^{b}$, Deirdre Pappalardo ${ }^{b}$, Oswin Baker ${ }^{c}$, Alex Christoupoulos ${ }^{c}$

\section{Affiliations}

a Section of Addictive Behaviour, Division of Mental Health, St George's, University of London, Cranmer Terrace, London SW17 ORE

b Kable Limited, The Courtyard, 55 Charterhouse Street, London EC1M 6HA

c MORI Social Research Institute, MORI House, 79-81 Borough Road, London SE1 1FY 
DH INFORMATION READER BOX

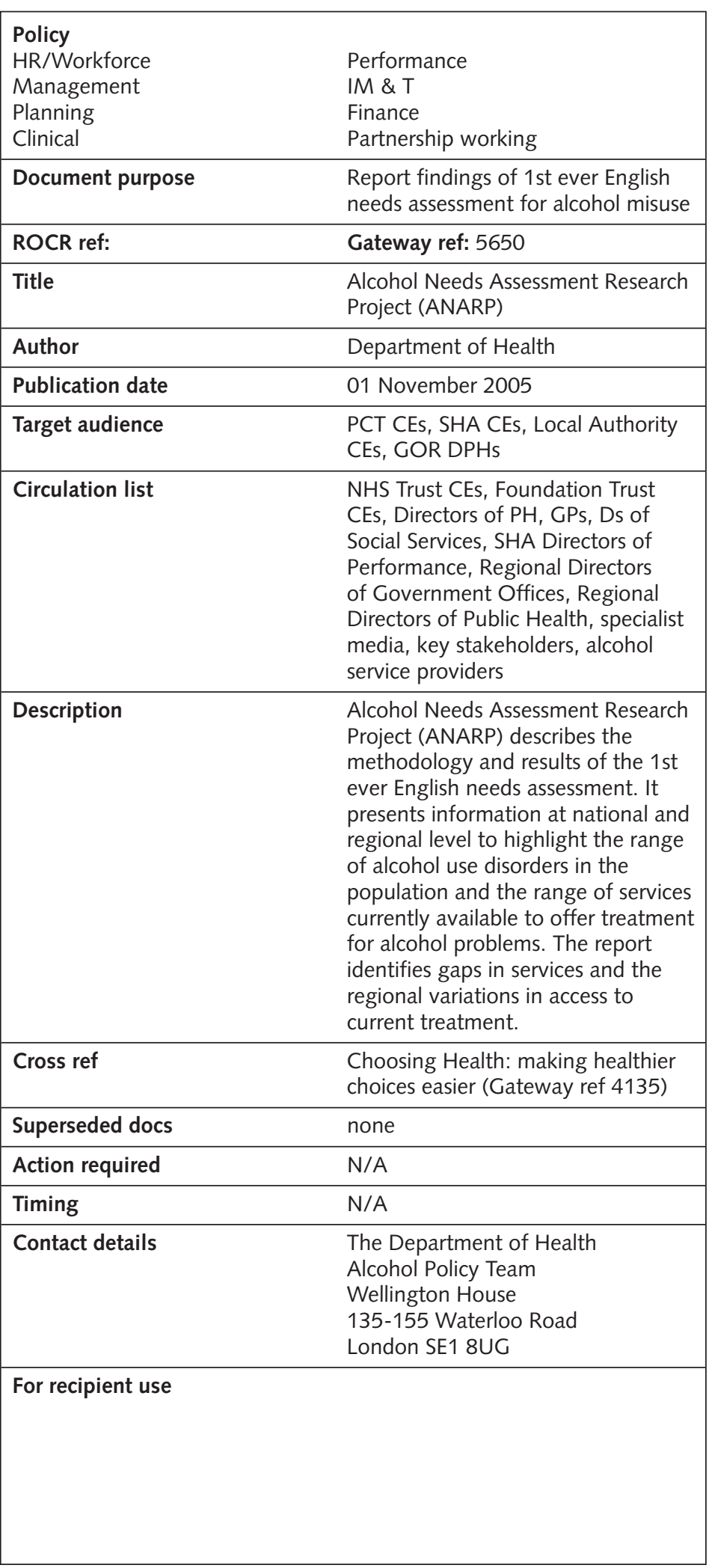




\section{Contents}

Foreword 2

Summary of findings 3

$\begin{array}{ll}\text { Background } & 5\end{array}$

$\begin{array}{ll}\text { Definitions } & 6\end{array}$

How the research was carried out $\quad 8$

Assessment of need for alcohol treatment in England 8

Identification of alcohol use disorders in primary care 10

General Practitioner \& Drug Action Team research 10

National survey of the provision of alcohol treatment services in England 10

Gap analysis 12

Key research findings 13

Assessment of need for alcohol treatment in England 13

Identification of alcohol use disorders in primary care 16

General Practitioner \& Drug Action Team research 17

National survey of the provision of alcohol treatment services in England 19

Gap analysis 21

Key conclusions of the research 23

Limitations of the research $\quad 25$

$\begin{array}{ll}\text { Implications for future research } & 28\end{array}$

$\begin{array}{ll}\text { Glossary of terms } & 29\end{array}$

$\begin{array}{ll}\text { Acknowledgements } & 30\end{array}$

$\begin{array}{ll}\text { References } & 31\end{array}$ 


\section{Foreword}

Alcohol misuse accounts for almost $10 \%$ of disease burden, surpassed only by tobacco and blood pressure. Up to 22,000 deaths each year are attributable to alcohol misuse and up to 150,000 hospital episodes. Alcohol misuse represents a major burden to the NHS and the wider health and social care systems.

I welcome this first ever national needs assessment concerning the levels of alcohol misuse amongst our population in England and the services available to respond to these needs. I hope it will assist in planning for the future and unlock thinking about developing approaches to address this national concern.

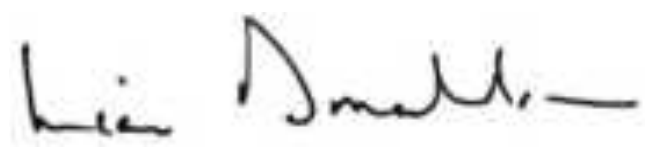

Sir Liam Donaldson

Chief Medical Officer 


\section{Summary of findings}

The Alcohol Needs Assessment Research Project (ANARP) is the first alcohol needs assessment in England conducted on a national scale.

The key findings are:

- The study found a high level of need across categories of drinkers. $38 \%$ of men and $16 \%$ of women (age 16-64) have an alcohol use disorder (26\% overall), which is equivalent to approximately 8.2 million people in England.

- There are $21 \%$ of men and $9 \%$ of women who are binge drinkers. There is a considerable overlap between drinking above 'sensible' daily benchmarks and 'sensible' weekly benchmarks for both men and women.

- The prevalence of alcohol dependence overall was $3.6 \%$, with $6 \%$ of men and $2 \%$ of women meeting these criteria nationally. This equates to 1.1 million people with alcohol dependence nationally.

- There was a decline in all alcohol use disorders with age. In relation to ethnicity, black and minority ethnic groups have a considerably lower prevalence of hazardous/harmful alcohol use but a similar prevalence of alcohol dependence compared with the white population.

- There is considerable regional variation in the levels of alcohol related need. The prevalence of hazardous/harmful drinking varied across regions from $18 \%$ to $29 \%$, whilst alcohol dependence varied between regions ranging from $1.6 \%$ to $5.2 \%$.

- The General Practice Research Database (GPRD) study found extremely low levels of formal identification, treatment and referral of patients with alcohol use disorders by general practitioners (GPs).

- GPs tended to under-identify younger patients with alcohol use disorders compared with older patients.

- The quantitative survey conducted with a random sample of $424 \mathrm{GPs}$ in England showed a higher level of GP awareness of alcohol use disorders than the GPRD research or previous surveys. 
- Although the majority (71\%) of patients with an alcohol use disorder identified by GPs were felt to need specialist treatment, the qualitative research suggested that many were not referred because of two main factors: perceived difficulties in access, with waiting lists for specialist treatment being the main reason given; and patient preference not to engage in specialist treatment.

- Both the qualitative and quantitative research identified a high level of satisfaction with specialist services once access was achieved.

- Drug Action Teams (DATs) receive funding from a range of sources including primary care trusts, local authorities and charitable funds. In the quantitative survey of DAT professionals, $86 \%$ of respondents said that their alcohol treatment budgets are much lower than drug budgets.

- Although not required to do so, $60 \%$ of DATs surveyed reported having a local alcohol strategy in place.

- In the qualitative research with DAT professionals, this group was aware of a 'very large gap' between the provision of alcohol treatment and need or demand, however it is expressed.

- The specialist alcohol agency survey successfully identified 696 agencies providing specialist alcohol interventions. This research has revealed $43 \%$ more agencies than identified in previous research.

- The mapping exercise identified considerable regional variation in the number of agencies, with London having the largest number of agencies and the North East the fewest.

- The largest proportion of referrals to alcohol agencies are self referrals (36\%) followed by GP/primary care referrals (24\%).

- The estimated annual spend on specialist alcohol treatment is $£ 217$ million. The number of whole time equivalent personnel working in specialist alcohol agencies across England is approximately 4,250.

- The average waiting time for assessment was 4.6 weeks. The shortest average wait for a region was 3.3 weeks and the longest wait was 6.5 weeks.

- The gap analysis estimated the number of alcohol dependent individuals accessing treatment per annum is approximately 63,000 , providing a Prevalence Service Utilisation Ratio (PSUR) of 18 (5.6\% of the in-need alcohol dependent population accessing alcohol treatment per annum or 1 in 18). 


\section{Background}

The Alcohol Harm Reduction Strategy for England ('Alcohol Strategy') (Prime Minister's Strategy Unit, 2004) identified a high level of alcohol misuse in society and consequent alcohol related harm. The Alcohol Strategy also notes that there has been insufficient focus on alcohol treatment in the past and that there is limited information on the extent to which existing levels of service provision meet demand. Following the publication of the Alcohol Strategy, a number of related projects have been initiated that will have an impact on planning of services by commissioners and providers and will support the Government's 'Programme of Improvement'. These projects include:

- the development of an alcohol service framework document, Models of Care for Alcohol Misusers (NTA, 2005), aimed at commissioners and providers of care;

- the development of an expert report on the effectiveness of alcohol health interventions;

- the commissioning of research into screening and brief interventions in primary health care and other settings; and

- the Alcohol Needs Assessment Research Project.

The Alcohol Needs Assessment Research Project (ANARP) was commissioned by the Department of Health, and was jointly conducted by St George's, University of London, Kable Ltd and MORI Social Research Institute. Its main focus was to measure the gap between the demand for and provision of specialist alcohol treatment services in England.

While there have been several local alcohol needs assessments in England, there has never been such an assessment conducted on a national scale. This needs assessment uses a 'systems' model to estimate access to specialist alcohol treatment rather than a 'historical demand' model as suggested by Rush (1990). A systems model estimates the gap between the prevalence of needs in the population and access to treatment, whereas a historical demand model simply estimates what is available. 


\section{Definitions}

For the purpose of this research, we have used the World Health Organization categorisation of alcohol use disorders which specifies three categories:

- Hazardous drinking: people drinking above recognised 'sensible' levels but not yet experiencing harm.

- Harmful drinking: people drinking above 'sensible' levels and experiencing harm.

- Alcohol dependence: people drinking above 'sensible' levels and experiencing harm and symptoms of dependence.

This categorisation is useful in assessing the level of need in the population and the gap between need and the provision of services appropriate to those needs. Different categories of drinker are likely to need different types of services.

For the purpose of this project 'need' is defined as the number of individuals in the general population with alcohol dependence who, if an alcohol intervention was available, could potentially benefit from this. This is derived from the one-year prevalence of alcohol dependence in the general population in England. This report focuses on the determination of the needs of people who are alcohol dependent rather than hazardous/harmful drinking in keeping with the project brief. However, where appropriate, reference is made to the prevalence of hazardous and harmful drinking.

'Service utilisation' or 'access' is defined as the number of individuals with alcohol dependence that access specialist alcohol treatment in a year. 'Demand' for alcohol intervention is a complex phenomenon that will be dependent on a range of individual, gatekeeper and specialist service-access factors. We have defined 'demand' in several ways in this study:

- The 'potential demand for health service resources' (PDHS) is the estimated number of individuals in England with alcohol dependence who have consulted their GP in a year. This is derived from GP consultation rates in the Psychiatric Morbidity Survey (PMS: Singleton et al., 2001). Clearly only a proportion of these individuals will be aware that they have an alcohol problem or would accept alcohol treatment if it were offered. However, this is the group in which there is a window of opportunity for identification and referral. 
- The 'potential demand for specialist alcohol services' (PDSA) is defined as the number of dependent drinkers per annum who access alcohol treatment in non-specialist services (e.g. medical or psychiatric facilities). Clearly not all dependent individuals who access non-specialist secondary care will have alcohol dependence as the presenting problem. However, they make significant demands on health service resources and could, if identified and appropriately referred, reduce their alcohol consumption and hence reduce the health consequences for their alcohol dependence. The PDSA is based on an estimate of the average proportion of dependent drinkers who received treatment in non-specialist treatment programmes (32\% over a four-year period; McAuliffe et al., 2001). A proportion of this group, if identified, could potentially be routed into specialist alcohol treatment.

- We defined 'actual demand for specialist alcohol services' (ADSA) as the number of dependent drinkers referred to alcohol services.

The 'gap' between need and service provision is expressed as the PrevalenceService Use Ratio (PSUR). The PSUR is the number in need of interventions divided by the number of people accessing specialist alcohol interventions. There is a need for consensus regarding the optimal level of PSUR for specialist alcohol services in England. The variation in PSUR across regions and demographic groups is particularly useful in identifying relative under-provision of access to services.

This research provides an estimate of the gap between need and provision at a national and regional level. Additional work will be undertaken to assist those responsible for the planning and delivery of alcohol services to match local need with provision more effectively. This will involve the development of tools to estimate the gap between need and provision at local level. 


\section{How the research was carried out}

The research consisted of eight related projects that were conducted in parallel over a period of six months between September 2004 and February 2005.

- A study of available data on the prevalence of alcohol use disorders in England, conducted by St George's.

- A study of the identification of alcohol use disorders in primary care using the General Practice Research Database, conducted by St George's.

- A quantitative telephone survey of GPs, conducted by Kable and MORI.

- Focus groups with GPs, conducted by Kable.

- A quantitative survey with Drug Action Team professionals, conducted by Kable and MORI.

- Focus groups with DAT co-ordinators, conducted by Kable.

- A national survey of alcohol treatment agencies in England, conducted by St George's and MORI.

- An analysis of the gap between need and service provision, conducted by St George's.

\section{Assessment of need for alcohol treatment in England}

The prevalence of alcohol use disorders in England and the need for alcohol services in each region was determined in an epidemiological profile using available data from general population figures in the 2001 Census, the 2000 Psychiatric Morbidity Survey (PMS: Singleton et al., 2001) and the 2001 General Household Survey (GHS: ONS, 2002). While alcohol use disorders clearly exist in the population aged $65+$ it was not possible to examine prevalence in this group to the necessary level of detail with the available data sets. Several measures of alcohol use disorders were examined to assess the need for alcohol services.

The estimate of alcohol need in English regions was based on a regression model that required the full data set to model predictors of alcohol use disorder prevalence. The PMS is the only available database to carry out this analysis, therefore the need estimates are primarily based on the Alcohol Use Disorders Identification Test (AUDIT) questionnaire (Saunders et al., 1993). The AUDIT 
questionnaire data from this survey was used to estimate the national and regional prevalence of hazardous and harmful drinking (AUDIT score 8-15) and alcohol dependence (AUDIT score $\geq 16$ ). The AUDIT has been found to have a high degree of sensitivity and specificity in detecting hazardous drinking in a UK population (Coulton et al., 2005). It is also known to possess high sensitivity and specificity when used as a dependence screen in the general population (Reinert and Allen, 2002; Rumpf et al., 2002). Furthermore the items on the AUDIT are anchored to the previous year's alcohol use pattern, making the scores a suitable measure of last year which can be matched to last year's service utilisation pattern in the gap analysis.

In this study, the cut-off score on the AUDIT of 16 is used to identify 'moderately and severely dependent' drinkers. Some, particularly the less dependent or less complex individuals, may either improve without referral to specialist services or by provision of less intensive specialised interventions either in primary or secondary care. Some of the population identified as 'hazardous and harmful' drinkers may not improve without receiving a more specialised intervention. It is not possible currently to determine exactly the size of these two subgroups. However, the reliability and robustness of using these standard cut-offs in the AUDIT outweigh these difficulties of interpretation. Supplementary data on alcohol quantity consumed were obtained from the 2001 General Household Survey, including measures of binge drinking (drinking more than eight units in one day in the past week for men and six units or more for women), hazardous drinking (drinking between 22 and 50 units per week for men and between 15 and 35 units per week for women) and harmful drinking (drinking more than 50 units per week for men and more than 35 units per week for women).

The analysis of the PMS data also provided hazardous and harmful drinking and alcohol dependence prevalence estimates for males and females, different age groups, and black and minority ethnic groups.

In order to estimate the need for any alcohol intervention in the population, the prevalence rates for alcohol related disorders were multiplied by the population size (16-64 years) in each region to produce the estimated number in need of alcohol services. Separate analyses were conducted for hazardous/harmful drinking and alcohol dependence.

In addition to using the PMS to provide estimates of alcohol need in the population, we also examined the PMS to develop a profile of alcohol related GP consultations in England. The PMS contained two items relating to consultation with a GP for a physical and/or mental health problem in the last year. Rates of GP consultation in individuals with alcohol related disorders were calculated to 
describe the volume of alcohol use disorder-related caseload expected in primary care, from which the estimated number of dependent drinkers that would represent a potential demand for health services could be calculated. This was achieved by applying multipliers derived from GP consultation rates in people with alcohol dependence to the estimated need for alcohol service figures.

\section{Identification of alcohol use disorders in primary care}

The research on the GPRD provided estimates of the level of identification of alcohol use disorders (hazardous/harmful drinkers and dependent drinkers) by GPs in England. The GPRD covers 480 general practices and 3.5 million patients across the UK. We selected data from England only. This was further broken down by gender, age groups and Government Office Regions (GOR).

In order to carry out the secondary analysis of the data from the GPRD, we identified Read Codes to classify the sample into the three categories of problems drinking (hazardous, harmful and dependent drinkers). The Read Codes were classified by experts in the alcohol field as being indicative of one of these three categories. Cases where we were not able to classify codes clearly into one of these categories were excluded. The study period was 12 months to provide oneyear prevalence estimates for alcohol use disorders in primary care.

\section{General Practitioner and Drug Action Team (DAT) research}

Four studies were conducted with GPs and DATs, including two quantitative surveys with a sample of GPs and DAT professionals to provide additional information regarding knowledge, attitudes and activities with individuals with alcohol use disorders and in relation to alcohol services. Two qualitative studies (focus groups) were also conducted with a sample of GPs and DAT co-ordinators to explore the issues in more depth.

\section{National survey of the provision of alcohol treatment services in England}

The national survey of alcohol treatment agencies in England enabled the estimation of the level of alcohol service utilisation (access) by alcohol dependent individuals. The survey was carried out in two main stages: a mapping stage and a survey stage. All statutory and non-statutory services in England that provided modalities of care for alcohol use disorders, both as 'primary' provision and as part of 'drug and alcohol' service provision, were identified through a comprehensive mapping process. In addition, those private sector services which received public funding for placements for alcohol use disorders through 'out of areas treatment' (OAT) 
requests or block contracts from publicly funded bodies were identified in the mapping stage. A survey tool designed to determine the nature of such provision was then sent out to all the publicly funded services identified as providing specialist treatment for AUDs.

The mapping stage was carried out by developing initially a comprehensive database that included service providers listed in directories and websites. All agency entries were validated by researchers, and contact details were checked. In addition, the research team approached managers from the National Drug Treatment Monitoring System (NDTMS) for their assistance in mapping alcohol agencies. All agencies identified by the regional managers were telephoned and those agencies which offered alcohol intervention were added to the database. A snowballing technique was then employed as the final check to ensure all agencies had been captured. Managers or senior clinicians from statutory services within each regional area were contacted by telephone to help determine whether there were any agencies not already included in the database.

A cross-sectional postal survey of alcohol treatment provision was developed by the research team. The survey was designed to identify the profile of services, measure the activity of alcohol agencies and examine inter-agency referrals. This was achieved by reviewing the literature regarding alcohol service utilisation and by consulting research teams involved in the National Assessment of Inpatient Drug Treatment and Residential Treatment Needs. This led to the formulation of two questionnaires: the Community Service Providers Questionnaire and Inpatient/Residential Service Providers Questionnaire. The questionnaires were peer-reviewed by experts in the field from statutory and non-statutory agencies and organisations. They were also piloted by disseminating the draft forms to a number of service providers for feedback and amendment.

The questionnaires were designed to collect data routinely collated by agencies. The data requested referred to the financial year 2003-04, and consisted of two sections. Section A considered the following areas: the nature of service provided; the sector; catchment area; primary role of service; summary activity data; staffing matrix; breakdown of referrals; breakdown of assessments; and modalities of treatment. Section B consisted of three open-ended questions that aimed to obtain qualitative data to explore the challenges faced by services in meeting the needs of clients, what groups may be poorly served and what resources may help services see and treat more people with alcohol problems.

The final questionnaire was sent to all eligible agencies in November 2004 by post, and non-responders were followed up with postal reminders and telephone calls. 
To facilitate the response rate, a telephone survey was conducted by MORI with the remaining non-responders in February 2005. This consisted of a reduced questionnaire, necessary to obtain activity information and some descriptive information about the agencies and their case mix.

\section{Gap analysis}

From the research described above, we were able to calculate the 'gap' between need, demand, and access for specialist alcohol services, expressed as the Prevalence Service Use Ratio (PSUR). The PSUR is the number in need of interventions divided by the number of people accessing specialist alcohol interventions. 


\section{Key research findings}

\section{Assessment of need for alcohol treatment in England}

The study found a high level of need across categories of drinkers based on a re-analysis of surveys conducted by the Office for National Statistics. Some 38\% of men and $16 \%$ of women (age 16-64) have an alcohol use disorder (26\% overall), which is equivalent to approximately 8.2 million people in England (source: PMS). Within this, $32 \%$ of men and $15 \%$ of women are hazardous or harmful alcohol users ( $23 \%$ overall) (see Figure 1). This equates to 7.1 million people in England. There are 21\% of men and 9\% of women who are binge drinkers, and there is a considerable overlap between drinking above 'sensible' daily benchmarks and 'sensible' weekly benchmarks for both men and women.

In relation to alcohol dependence, we found that the overall prevalence of alcohol dependence was $3.6 \%$, with $6 \%$ of men and $2 \%$ of women meeting these criteria nationally (see Figure 1). This equates to 1.1 million people with alcohol dependence nationally.

Figure 1: Percentage of people in England with an alcohol use disorder, broken down by category of problem drinking and gender

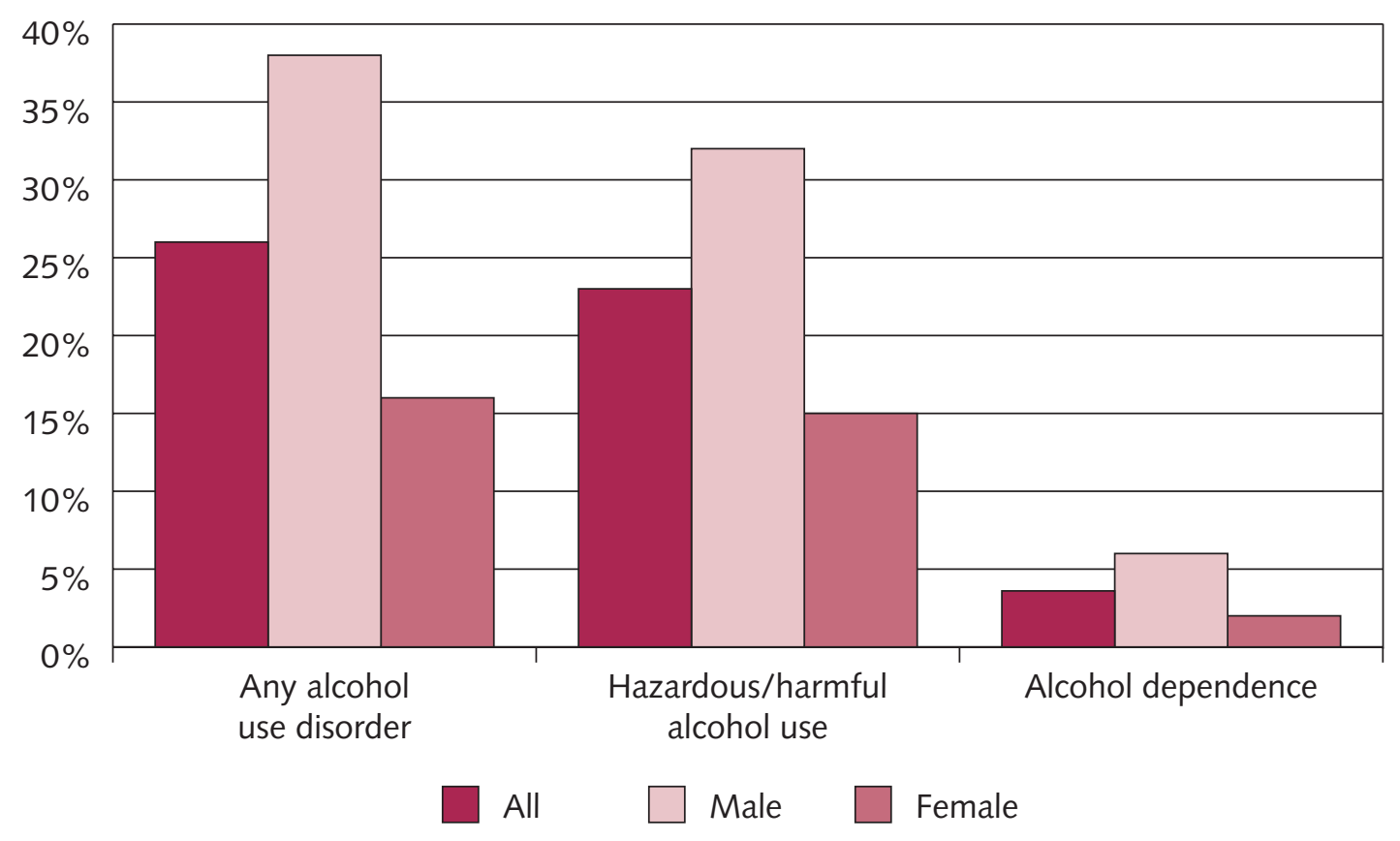


Estimates of prevalence of hazardous and harmful drinking obtained using the AUDIT questionnaire are similar to estimates of prevalence of those exceeding 'sensible' weekly units (as in the General Household Survey). Estimates of prevalence of people exceeding 'harmful' levels of weekly alcohol intake ( $>50$ units/week in men and $>35$ units/week in women) are similar to estimates of people experiencing alcohol dependence (as in the PMS). We therefore do not find a good justification for separating drinkers into three categories so we have used a two-category classification for this research project, with the categories of 'hazardous/harmful drinkers' and 'dependent drinkers'.

There was a decline in all alcohol use disorders with age, with younger people showing a higher prevalence of both hazardous/harmful drinking and alcohol dependence than older people (see Figure 2)

Figure 2: Percentage of people in England with an alcohol use disorder by age

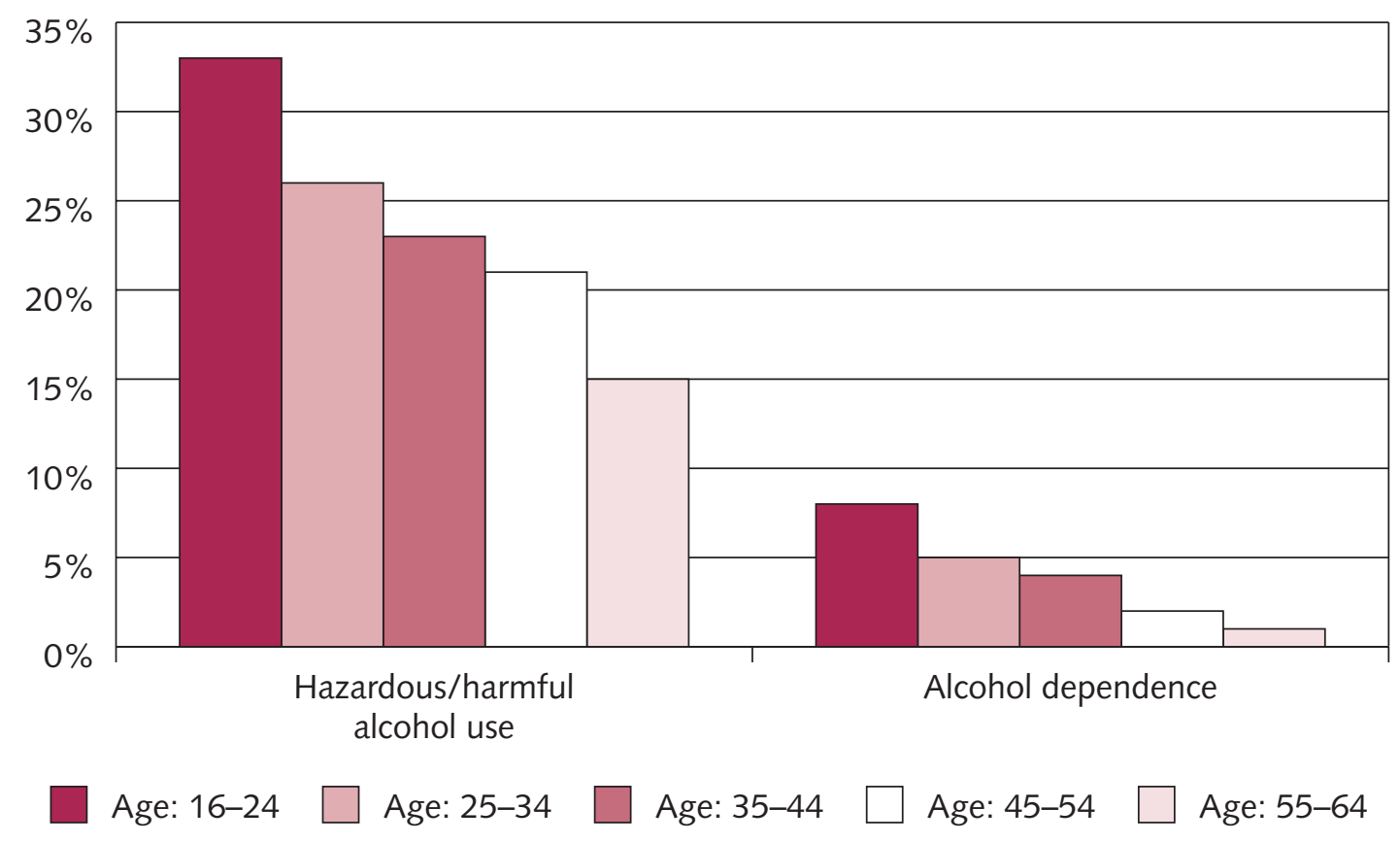

In relation to ethnicity, black and minority ethnic groups have a considerably lower prevalence of hazardous/harmful alcohol use, but a similar prevalence of alcohol dependence compared with the white population (see Figure 3). 
Figure 3: Percentage of people in England with an alcohol use disorder by ethinicity

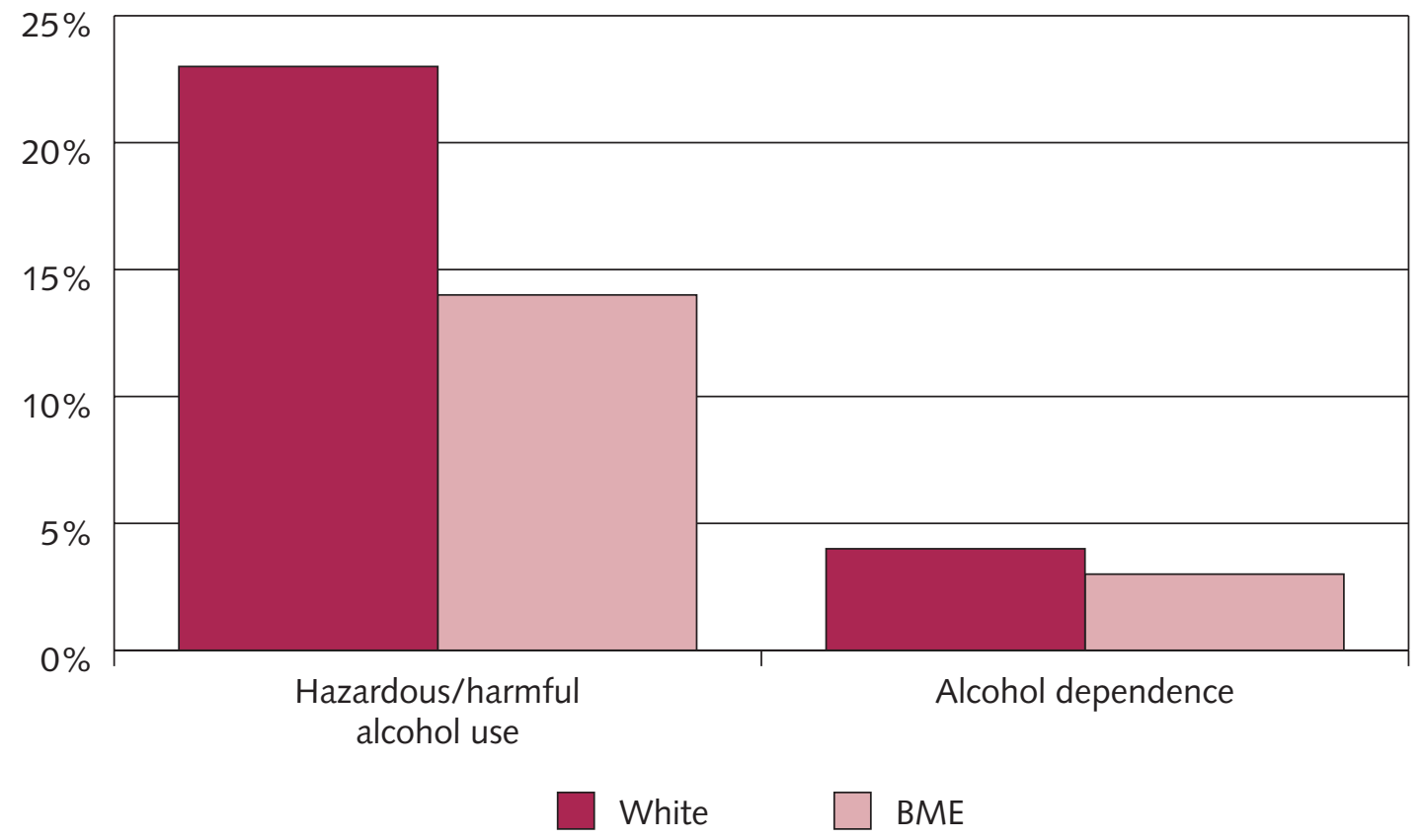

There is considerable regional variation in the levels of alcohol related need. The prevalence of hazardous/harmful drinking varied across regions from 18\% to $29 \%$ (see Figure 4), but also with some differences between men and women. There was also considerable variation in levels of alcohol dependence between regions, ranging from $1.6 \%$ to $5.2 \%$. Interestingly, the regions with the highest prevalence of hazardous/harmful drinking are different from those with the highest prevalence of alcohol dependence. This suggests the need for different levels/types of services in different regions. 
Figure 4: Percentage of people in England with an alcohol use disorder by region

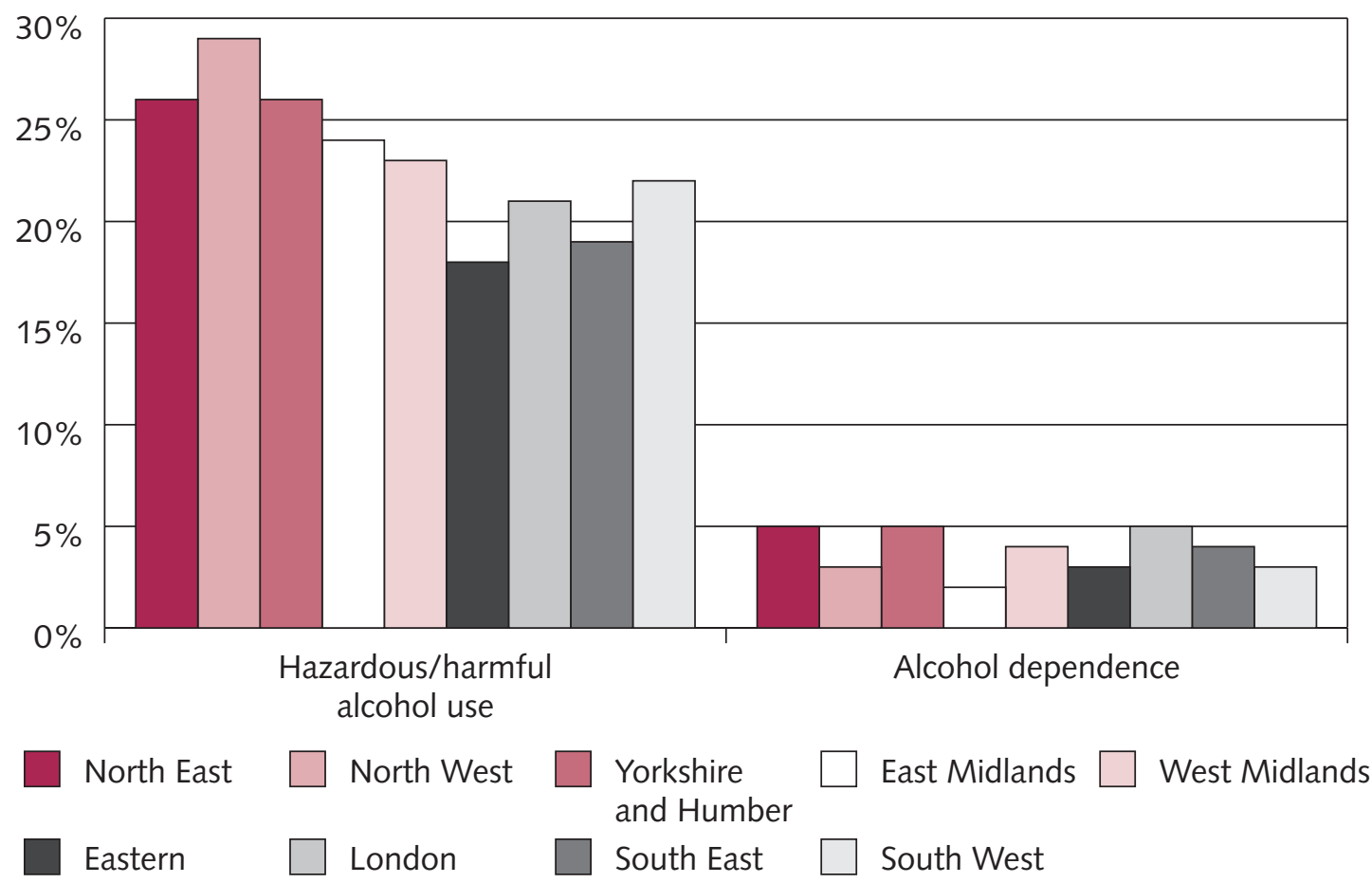

\section{Identification of alcohol use disorders in primary care}

The GPRD study found extremely low levels of formal identification, treatment, and referral of patients with alcohol use disorders by GPs. After adjusting for differences in consultation rates between groups of people with alcohol use disorders, GPs identified in their database entries approximately 1 in 67 male and 1 in 82 female hazardous/harmful drinkers. The identification rate for alcohol dependence was 1 in 28 and 1 in 20 for males and females respectively.

GPs tended to under-identify younger patients with alcohol use disorders compared with older patients, which could not be accounted for by differences in consultation rates. There was also considerable regional variation in the identification of alcohol use disorders, with Yorkshire and Humber and London having the lowest identification rates for both hazardous/harmful drinking and dependence. GPs in these regions were three to four times less likely to identify alcohol use disorders than in the best performing regions. 


\section{General Practitioner and Drug Action Team research}

\section{GP research}

The quantitative survey conducted with a random sample of 424 GPs in England showed a higher level of GP awareness of alcohol use disorders than the GPRD research or previous surveys. The findings were that $55 \%$ of GPs reported seeing between one and five patients with alcohol dependence in the previous month (mean 8.1/month) and a mean of 14.2 patients who were excessive drinkers, providing an overall estimate of 22.2 alcohol use disorders identified per month. This is about five times the level of identification found in our previous national survey and stands in contrast to the GPRD results. A high proportion of GPs reported the use of systematic screening methods and the provision of a wide range of in-house interventions.

Although the majority (71\%) of alcohol use disorder patients identified by GPS were felt to need specialist treatment, the qualitative research suggested that many were not referred because of two main factors: perceived difficulties in access, with waiting lists for specialist treatment being the main reason given; and patient preference not to engage in specialist treatment. Both the quantitative and the qualitative research suggested that GPs limit the 'demand' for specialist services by low levels of routine enquiry about alcohol issues and by finding alternatives to specialist treatment such as in-house interventions and referral to self-help groups such as Alcoholics Anonymous (AA).

The qualitative research findings indicate that in-house interventions and referral to self-help groups are mechanisms for coping with the perceived difficulties in access to specialist treatment, but the process of this is not informed by systematic criteria. The existing mutual aid groups in the UK may play an important role in addressing the problem of alcohol related harm by providing a possible solution to the problem of the gap between the demand for and the perceived accessibility of services. Expanding these groups beyond AA to include different approaches, and to involve more scientifically validated principles, may widen the use of such groups by the in-need population.

Both the qualitative and quantitative research identified a high level of satisfaction with specialist services once access was achieved. There was also a perceived high level of 'shared care' and a high level of satisfaction with support provided by specialists compared with previous research. It is possible that initiatives in the development of shared care for drug misusers may have had a 'halo' effect on alcohol treatment, given that many addiction services, particularly in the NHS, are combined drug and alcohol services. 
GPs generally welcomed the possibility of more training in alcohol issues, which provides potential support for other Department of Health initiatives in primary care. GPs also expressed a preference for in-person training rather than internet or other forms of distance learning.

\section{DAT research}

Quantitative research with 80 DAT professionals and 6 qualitative focus groups provided information on alcohol services and need from a commissioning perspective.

DAT professionals reported that demand for alcohol services is generally greater than supply, and that budgets for alcohol services are generally lower than for drug services, although they perceived the problems associated with alcohol to be considerably greater.

Of the DATs who responded the most widely reported alcohol services available were counselling $(81 \%)$, inpatient detoxification $(63 \%)$, specialist advice and information (60\%) and day treatment (53\%). The groups identified as being particularly disadvantaged in terms of access to alcohol treatment were black and ethnic minority groups $(19 \%)$, women $(14 \%)$, rural communities $(16 \%)$, homeless people $(11 \%)$ and asylum seekers/refugees $(10 \%)$. Approximately $30 \%$ of DAT staff did not identify any particular disadvantaged groups.

Funding for alcohol services comes from a range of sources including primary care trusts, local authorities and charitable funds. There was considerable variation in the funding available for alcohol services across DAT areas, ranging from $£ 35,000$ to $£ 8$ million. Some $86 \%$ said that their alcohol treatment budgets are much lower than their drug budgets.

Although not required to do so, $60 \%$ of DATs surveyed reported having a local alcohol strategy in place. Some $53 \%$ of DAT organisations reported having plans to change the level or mix of provision of alcohol treatment services within the next 12 months; however, when asked to elaborate, plans tended not to be detailed or specific and often lacked specific funding to implement them.

In the qualitative research with DAT professionals, this group was aware of a 'very large gap' between the provision of alcohol treatment and need or demand, however it is expressed. They also believed that the harm resulting from alcohol misuse is far greater to both the individual and the community than the harm resulting from drug misuse. 
Concerns were expressed about the inclusion of alcohol in the remit of DATs or Drug and Alcohol Action Teams, unless it is supported by sufficient funding; otherwise it would be difficult to meet drug targets.

There was a concern that the supply of alcohol needed to be tackled as much as demand, and that the alcohol strategy did not have robust measures to do this. Another concern was that the alcohol strategy focuses more on reducing crime and disorder rather than addressing health and social care needs.

DAT professionals expressed concern about a delay in action to improve services following publication of the alcohol strategy. This has had a negative impact on professionals in the field who feel the strategy has raised expectations but has not produced the desired impact on services on the ground.

DAT professionals also reported that the lack of specific targets in the alcohol strategy will hamper local action to develop alcohol initiatives.

\section{National survey of the provision of alcohol treatment services in England}

The specialist alcohol agency survey successfully identified 696 agencies providing specialist alcohol interventions. As a result of an extensive national service mapping exercise, this research has revealed $43 \%$ more agencies than were previously identified. We anticipate that this new database will be useful for service planning and the provision of information on the availability of treatment agencies.

The mapping exercise identified considerable regional variation in the number of agencies, with London having the largest number of agencies and the East Midlands with the fewest (see Figure 5). 
Figure 5: Distribution of alcohol treatment agencies identified by ANARP mapping exercise by region $(n=696)$

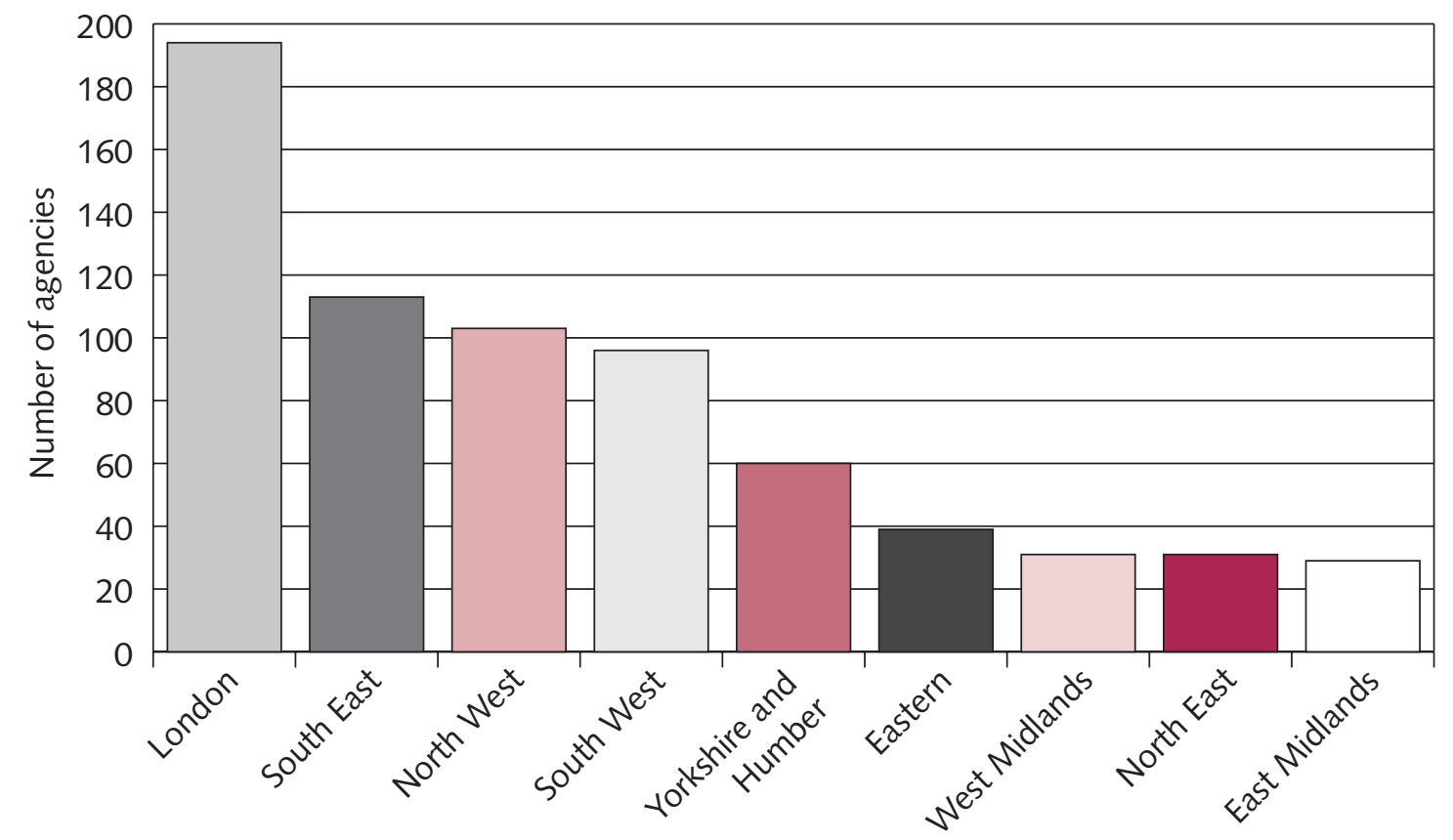

The overall response rate to the survey was $56 \%$. Nearly $69 \%$ of alcohol agencies were community based, and $31 \%$ were residential services. One-third were primarily alcohol services and 58\% were combined drug and alcohol services. Agencies primarily treating drug misusers that also provided services to alcohol misusers accounted for $7 \%$ of all agencies. Over half of all agencies were nonstatutory (voluntary), one-third statutory (NHS) and $8 \%$ private sector.

Community agencies most commonly provided advice, brief interventions and structured psychological interventions. Residential agencies most commonly provided residential rehabilitation and inpatient treatment, including detoxification. Overall, $45 \%$ of community agencies and $46 \%$ of residential agencies provided alcohol detoxification.

We found $91 \%$ of clients attending residential agencies were reported to be alcohol dependent compared with $71 \%$ of community agency clients. In both types of agency, clients with more severe alcohol dependence were the largest group of clients.

The largest proportion of referrals to alcohol agencies are self-referrals (36\%), followed by GP/primary care referrals (24\%). Some $8.2 \%$ of referrals to alcohol agencies are from another alcohol agency, pointing to the potential for double counting of clients in needs assessment. 
The estimated annual spend on specialist alcohol treatment is $£ 217$ million. It should, however, be noted that this estimate is based on a number of assumptions described in 'Limitations of the research' below. The estimated number of wholetime equivalent personnel working in specialist alcohol agencies across England is approximately 4,250 .

The average waiting time for assessment was 4.6 weeks (4.1 and 5.5 for community and residential agencies respectively). The shortest wait was observed in the South East region (3.3 weeks) and the longest wait was in the North East region (6.5 weeks). The average number of patients (monthly) on the waiting list for assessment was 15.2 (18 for community agencies and 9 for residential agencies) and again showed regional variations. Waiting list length was positively correlated with both the number of staff and the number of referrals.

The qualitative responses obtained in the survey revealed that agency staff perceived the main challenges and issues facing services to be focused around a lack of funding, a lack of resources, and the focus on drug services compared with alcohol. The groups that are most commonly perceived to be poorly served by alcohol treatment services are women caring for children, homeless people, ethnic minorities and clients with dual diagnosis. All clients with alcohol problems were, however, often perceived as being poorly served. The resources that alcohol agency staff feel would help to improve services in particular are more suitably qualified staff, better access to detoxification/rehabilitation services, and more funding for services.

\section{Gap analysis}

The gap analysis estimated the number of alcohol dependent individuals accessing treatment each year. Using the data obtained from the survey of alcohol treatment agencies in England, this was estimated to be approximately 63,000 individuals, providing a PSUR of 18 . This equates to $5.6 \%$ of the 'in need' alcohol-dependent population accessing alcohol treatment each year. Women were 1.7 times more likely to access alcohol treatment than men (PSUR women: 12; men: 21). There was considerable regional variation in PSUR from 1 in $102(1 \%$ of alcohol dependent people accessing treatment in a year) in the lowest access region (North East) to 1 in 12 (8\% of alcohol dependent people accessing treatment in a year) in the highest (North West) (see Figure 6). The gap analysis also highlighted that more than twice as many people are referred for alcohol treatment than those who actually access treatment (2.7:1). 
Figure 6: Prevalence Service Utilisation by region

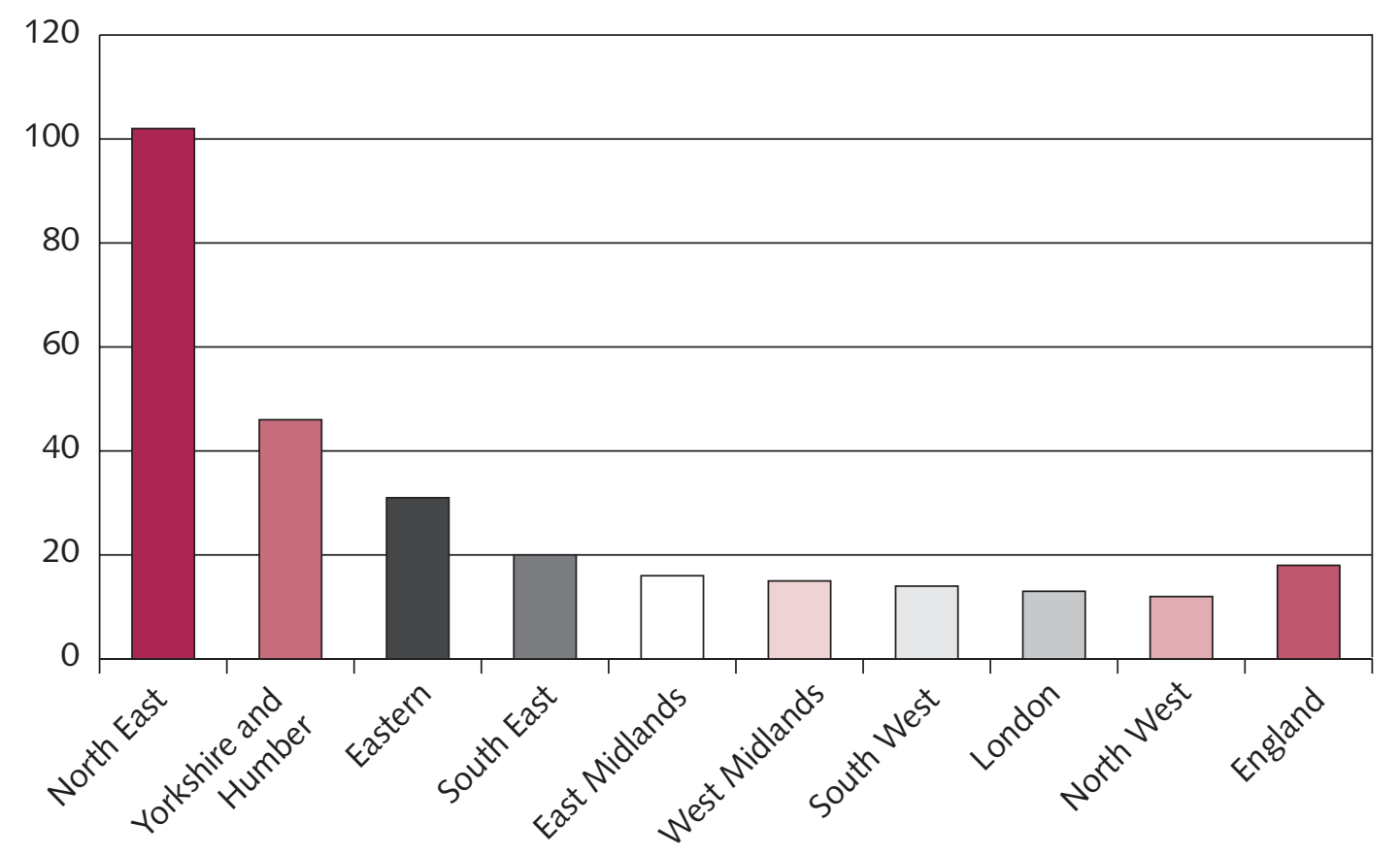




\section{Key conclusions of the research}

There is a large gap between the need for alcohol treatment and actual access to treatment with only approximately 1 in $18(5.6 \%)$ alcohol dependent individuals accessing specialist alcohol treatment nationally per annum. In North America, an access level of 1 in $10(10 \%)$ alcohol dependent individuals entering treatment per annum is regarded as a 'low' level of access, 1 in 7.5 (15\%) 'medium' and 1 in 5 (20\%) 'high' (Rush, 1990). There is a need for consensus on the optimal level of access which should be achieved in England

This gap between need and access is greater than some previous studies assessing alcohol need. This can be partly accounted for by the different methodologies used. This estimate is based on more direct measures of need and access than is the case in some previous studies, and therefore represents a more robust measure of gap.

The evidence of a shortfall in provision is further reinforced by the additional finding of a large, nearly ten-fold, regional variation in PSUR for alcohol dependence. This will be a particularly powerful tool for local areas to use in considering the adequacy of their current provision.

Approximately a third of alcohol dependent individuals referred to treatment actually access treatment. This suggests that there is considerable potential to increase engagement with services through a variety of means. As $36 \%$ of referrals to alcohol specialist services are self-referrals, this points to the potential need for initiatives to improve access, including better information for the public on treatment that is available. However, consideration also needs to be given to the capacity of specialist treatment services to meet any increase in demand generated by such initiatives.

A large number of individuals with alcohol dependence consult their GP annually (at a higher rate than moderate drinkers) and a large proportion access general medical and psychiatric services, not necessarily with alcohol dependence as the primary presenting problem.

The quantitative survey of GPs revealed a considerably higher level of identification of people with alcohol use disorders than either the GPRD study or previously published UK research. GPs in this study reported identification of 
nearly five times that found in a previous national survey. This disjunction between the GPRD results and the telephone survey results requires explanation. This may be a reflection of response bias. Alternatively, there may be a higher level of awareness of patients with alcohol problems than the numbers that are actually recorded or referred to specialist care, particularly in view of the qualitative research conducted with GPs. This may be because GPs are loath to record alcohol diagnoses or are perhaps, as the qualitative research suggests, reluctant to refer patients to services that are perceived as overstretched. Further research is required to explore these possibilities.

The results of this study also showed higher levels of reported screening and intervention activity in primary care than previous research and a higher level of confidence in dealing with alcohol problems. Nevertheless, GPs expressed the need to have more training in dealing with alcohol in primary care.

There was a high level of satisfaction with specialist services among GPs and a high level of shared care, but also a high level of difficulty in accessing treatment, with waiting lists/times being the main barrier. It is therefore likely that increased capacity in alcohol services would lead to increased actual demand and access.

There is clearly considerable scope for increased identification and referral to specialist care from generic services including primary care, general hospitals, mental health services, criminal justice agencies and social services. These findings suggest this needs to be tempered by ensuring adequate capacity in specialist alcohol services to meet increased demand from initiatives to increase screening and referral activity in non-specialist services. 


\section{Limitations of the research}

The research on service provision in primary care excluded practice nurses and other professionals working in that setting. While it is acknowledged that these providers have an important role in managing alcohol problems, their inclusion was not feasible within the scope and timescale of the current study. There is a need to examine the role of healthcare professionals, in general, in managing alcohol dependence in primary care settings and quantify the extent of their therapeutic involvement. Future studies should address this limitation.

This study focused on the gap in service provision for persons with alcohol dependence. Consideration for hazardous/harmful drinkers in the same depth would have improved the comprehensiveness of the study implications, and provided a higher estimate of the size of the population in need of some form of intervention. However, this additional work was beyond the remit of the current project, which was to determine the needs of people who are alcohol dependent and their access to specialist treatment.

It is likely that most hazardous and harmful drinkers will require information, advice or brief interventions in primary care or other generic settings. There is also a broad assumption in this study that most dependent drinkers who could benefit from alcohol intervention will require specialist alcohol treatment. However, this study was not designed to assess the scale and requirements for care of the 'more in-need' hazardous/harmful drinkers, and the requirements for the 'less in-need' dependent drinkers. In particular, it is likely that some hazardous/harmful drinkers will need more specialised interventions in primary care or in specialist alcohol services. Also, some dependent drinkers will be likely to respond to less intensive, specialised interventions in secondary care or, if available, through primary care, or they may respond to brief interventions in primary care. It is important to acknowledge this limitation, which is often dealt with in practice by using a 'stepped care' approach as proposed in Models of Care for Alcohol Misusers (NTA, 2005). This study should not be interpreted to suggest a simple dichotomy of types of provision.

Another related limitation is the focus on specialist services in the gap analysis, with little consideration for generic services that often provide some intervention for people with alcohol dependence. Again, the primary objective was to identify gaps in existing provision dedicated to alcohol dependence. It is often the case that mutual aid groups provide some level of service to alcohol dependent 
persons. Given the anonymity associated with many of these groups, it is difficult to quantify the extent of their contribution to service provision. Therefore, including them in this study would have added little information for estimating gap in service provision.

Our estimate of service utilisation is also based on several assumptions including, importantly, extrapolation from data provided by agency responders (56\%) to the non-responders. However, the use of linear regression modelling has allowed adjustment for differential regional response rates and service activity, which provide a more realistic estimate of true service utilisation. We have also adjusted the access rates for the proportion of individuals being referred between alcohol agencies $(8.2 \%)$ to avoid double counting of individuals, and we have only looked at the proportion of individuals referred to and accessing treatment that are reported to have alcohol dependence. This clearly assumes that agencies are able to provide a valid assessment of alcohol dependence. Nevertheless, the fact that more individuals are reported to have moderate to severe alcohol dependence in residential agencies compared with community agencies suggests that there is a reasonable degree of validity in this assumption. We have assumed that our agency mapping exercise captured all specialist alcohol treatment providers in England. This may not be the case, but we have identified a significantly larger proportion of agencies than was previously known to provider databases or previous agency surveys. Therefore, we are confident that this survey provides the best estimate of alcohol treatment agencies in England so far.

The estimated annual spend on specialist alcohol treatment in England in this report is based on a number of assumptions. Because many agencies in the survey are combined drug and alcohol agencies, and it was not possible to identify the proportion of spend specifically on alcohol treatment, we based our estimate on those agencies identified as primarily alcohol agencies and extrapolated the expenditure to combined agencies based on this. Our estimate is higher than the Government's previous estimate of $£ 95$ million (PMSU, 2004). Although this previous estimate was based on a smaller number of agencies, our estimate is still higher after taking this into consideration. We suspect that our estimate is larger than the actual spend as it was heavily influenced by combined drug and alcohol agencies, and it was not possible to identify the relative spend on alcohol and drugs work in these agencies. Further work is needed to identify the relative spend on alcohol versus drugs in combined drug and alcohol agencies to refine our estimate of treatment spending. 
We have also assumed that all individuals accessing treatment in a given region are resident in that region as we have not been able to study the regional source of referral data in this project. Clearly, it is possible that some referrals, particularly to residential rehabilitation programmes, are from outside the region in which the agency is located.

We have also assumed that all individuals who accessed specialist alcohol treatment actually received an appropriate intervention. We have made no adjustment (as did Rush, 1990) for the number of individuals who drop out of treatment before completion, as we have no data on this from the survey. Clearly, individuals with more severe alcohol dependence are more likely to require detoxification and more structured care than less severely dependent drinkers and hazardous and harmful drinkers. Less than half of community and residential agencies provide facilities for detoxification (or assisted alcohol withdrawal). Therefore, the level of access to detoxification is likely to be less than the level of access to any alcohol treatment services. Further exploration of this issue and the extent to which presenting needs are actually met by specialist alcohol agencies, will need a more in-depth study of treatment pathways in local areas rather than the more global picture provided by this study.

Our study did not examine the PSUR for hazardous and harmful alcohol use. Our data shows that a minority of individuals accessing specialist alcohol treatment are hazardous and harmful drinkers. However, it is possible that a larger number of these individuals could benefit from a specialist alcohol intervention than has been captured by this study. This requires further consideration, including the relevant care pathways for hazardous and harmful drinkers.

The data available from this project cannot be used alone in determining local need or gaps in provision for specialist alcohol services. The data does provide a national and regional picture of need for and access to specialist alcohol treatment, an important foundation upon which to analyse local needs more fully. Additional work will be undertaken to assist in providing local estimates of the gap between need for and provision of alcohol treatment at a local level. 


\section{Implications for future research}

This study has identified several areas where further research is needed.

Future research involving better prospective data collection of prevalence of alcohol use disorders, both in the general population and in primary and secondary care (including general practice, accident and emergency departments, and general hospitals) would be useful in assessing the impact of initiatives to combat alcohol misuse. In addition, routine collection of data on people with alcohol use disorders attending specialist treatment services would allow ongoing assessment of access to treatment in 'real time'.

Research would also be useful to study the relationship between indicators of alcohol related harm and the prevalence of alcohol use disorders in the general population. This would provide a better understanding of methods to assess alcohol related need and the impact of alcohol policies in the future.

The inclusion of items regarding alcohol help-seeking in future Psychiatric Morbidity Surveys/General Household Surveys is needed, including questions on whether individuals have tried to access treatment unsuccessfully, or would access treatment if it was available. This would provide a more direct measure of 'demand' for alcohol treatment than has been possible in this study.

More in-depth analysis of treatment pathways for both alcohol dependent and hazardous/harmful drinkers is required. This should help to provide more detail on the need for and access to alcohol treatment, as well as barriers to access.

Research is needed to examine the best methods of increasing the level of screening, identification, intervention and referral of both alcohol dependent and hazardous/harmful drinkers attending primary and secondary care, and criminal justice and social care agencies. This should include study of the impact of screening initiatives on demand for specialist alcohol treatment.

Ways to improve methods of engaging and retaining people with alcohol dependence in treatment should be studied, including methods such as assertive outreach and intensive case management. Research should also examine the effects of these methods on drinking behaviour, health and cost outcomes. Again, issues surrounding the impact of such initiatives on treatment capacity should be addressed, given that increasing the number of people who enter or remain in treatment will affect capacity. 


\section{Glossary of terms}

AA - Alcoholics Anonymous

ADSA - actual demand for specialist alcohol services

ANARP - Alcohol Needs Assessment Research Project

DAT - Drug Action Team

DAAT - Drug and Alcohol Action Team

DH - Department of Health

GHS - General Household Survey

GOR - Government Office Regions

GP - - General Practitioner

GPRD - General Practice Research Database

NHS - National Health Service

PCT - primary care trust

PDHS - potential demand for health service resources

PDSA - potential demand for specialist alcohol services

PMS - Psychiatric Morbidity Survey

PSUR - Prevalence Service Utilisation Ratio 


\section{Acknowledgements}

We would like to thank the Department of Health for supporting this research. We would also like to thank the General Practice Research Database, the Office for National Statistics and Alcohol Concern for their help and providing essential information for this project. We also give thanks to all the GPs, DAT professionals and specialist agencies for taking part in the various surveys and focus groups. A great deal of professional time was given at very short notice by many people to help with this project, and for that, we are extremely grateful. 


\section{References}

Coulton, S., Drummond, C., James, D., Godfrey, C., Parrott, S. and Peters, T. (on behalf of the Stepwice Research Team) (2005) Opportunistic Screening for Alcohol Use Disorders in Primary Care: A comparison of screening methods, submitted.

McAuliffe, W., Dunn, R. and Zhang, C. (2001) An Integrated Substance Abuse Treatment Needs Assessment for North Dakota: Final report. Cambridge, MA, North Charles Planning Group.

National Treatment Agency (2005) Models of care for alcohol misusers. Available online at: http://www.nta.nhs.uk/programme/guidance/mocam.html

Office for National Statistics (2002) Living in Britain: Results from the General Household Survey 2001. London, The Stationery Office.

Prime Minister's Strategy Unit (2004) Alcohol Harm Reduction Strategy for England, London, PMSU.

Reinert, D.F. and Allen, J.P. (2002). The Alcohol Use Disorders Identification Test (AUDIT): A review of recent research. Alcoholism: Clinical and Experimental Research, 26: 272-9.

Rumpf, H-J., Hapke, U., Meyer, C. and John, U. (2002). Screening for alcohol use disorders and at-risk drinking in the general population: Psychometric performance of three questionnaires. Alcohol and Alcoholism, 37: 261-8.

Rush, B. (1990) A systems approach to estimating the required capacity of alcohol treatment services. British Journal of Addiction, 85: 49-59.

Saunders, J.B., Aasland, O.G., Babor, T.F., De la Fuente, J.R. and Grant, M. (1993) Development of the Alcohol Use Disorder Identification Test (AUDIT): WHO collaborative project on early detection of persons with harmful alcohol consumption. Addiction, 88: 791-804.

Singleton, N., Bumpstead, R., O'Brien, M., Lee, A. and Meltzer, H. (2001) Psychiatric Morbidity among Adults living in Private Households, 2000. London, Office for National Statistics/HMSO. 


\section{(c) Crown copyright 2005}

Produced by COI for the Department of Health

271230 1p 2.5k Nov05 (CWP)

If you require further copies of this title quote 271230/Alcohol Needs Assessment Research Project (ANARP): The 2004 national alcohol needs assessment for England and contact:

DH Publications Orderline

PO Box 777

London SE1 6XH

Tel: 08701555455

Fax: 01623724524

E-mail: dh@prolog.uk.com

(2) 08700102870 - Textphone (for minicom users) for the hard of hearing

$8 \mathrm{am}$ to $6 \mathrm{pm}$ Monday to Friday.

271230/Alcohol Needs Assessment Research Project (ANARP): The 2004 national alcohol needs assessment for England may also be made available on request in Braille, on audio-cassette tape, on disk and in large print. 